Portland State University

PDXScholar

$5-24-2019$

\title{
Interactions of Phosphatidylcholine Vesicles and Citrate-stabilized Gold Nanoparticles
}

Marie Vallens

Portland State University

Follow this and additional works at: https://pdxscholar.library.pdx.edu/honorstheses

Let us know how access to this document benefits you.

\section{Recommended Citation}

Vallens, Marie, "Interactions of Phosphatidylcholine Vesicles and Citrate-stabilized Gold Nanoparticles" (2019). University Honors Theses. Paper 692.

https://doi.org/10.15760/honors.710

This Thesis is brought to you for free and open access. It has been accepted for inclusion in University Honors Theses by an authorized administrator of PDXScholar. Please contact us if we can make this document more accessible: pdxscholar@pdx.edu. 


\title{
Interactions of phosphatidylcholine vesicles and citrate-stabilized gold nanoparticles
}

\author{
by \\ Marie Vallens
}

An undergraduate honors thesis submitted in partial fulfillment of the

\author{
requirements for the degree of \\ Bachelor of Science
}

in

University Honors

and

Biomedical Physics

Thesis Adviser

Drake C. Mitchell, Ph.D.

Portland State University

May 24, 2019

Revision Date: June 03, 2020 


\begin{abstract}
Citrate-coated gold nanoparticles (AuNPs) are increasingly implemented in many biomedical applications due to their novel electrochemical properties and further stabilization against influence from the cellular environment by conjugation of citrate ligands to the exterior of the gold nanocrystalline lattice. AuNPs have the ability to permeate the cell membrane without disturbing the nucleus, with some size regimes, without producing any known toxic effects. This work presents a collection and analysis of time-resolved fluorescence spectroscopy data describing the interior of the bilayer of unsaturated 16:0 - 18:1 phosphatidylcholine large unilamellar vesicles when exposed to 5, 10, and 20 [nm]-diameter citrate-capped AuNPs at physiological temperature. A description of how the membrane was affected at the molecular level was formulated by analyzing frequency-domain lifetime and time-resolved fluorescence anisotropy decay measurements of AuNP-phosphatidylcholine assemblies.
\end{abstract}

KEYWORDS: Gold nanoparticles, large unilamellar vesicles, POPC, phosphatidylcholine, frequency-domain lifetime, time-resolved fluorescence anisotropy 


\section{Background}

Mammalian biomembranes typically consist of hundreds of lipid species and proteins ( $25 \%$ to $65 \%$ protein by weight) and are abundant in phospholipids and cholesterol. ${ }^{1} \mathrm{~A}$ range of different phospholipid classes exist within humans, of which phosphatidylcholine (PC) is one of the most prevalent, and as such, is one of the more documented. ${ }^{2}$ Additionally, PC biomolecules contribute to membrane-mediated operations within the cell, including enzyme activation and cell signaling.

Conditions in the interior of the membrane bilayer dictate the efficacy of membrane functions. Most mammalian cell barriers are composed of a semi-permeable amphiphilic phospholipid bilayer plasma membrane with hydrophilic head groups oriented on the exterior of the bilayer, and hydrophobic acyl groups oriented in the interior of the bilayer. ${ }^{3}$ Previous studies have demonstrated ready diffusion of AuNPs through this membrane reportedly due to their size and surface chemistries. ${ }^{4} 5$ Furthermore, gold nanoparticles have increased stability after forming surface bonds with citrate. ${ }^{6}$ A widely used method for synthesis of citrate-stabilized AuNPs is the citrate method, which coats the gold nanocrystal and produces uniform nanoparticles tunable in diameter in range of $\sim 10-100[\mathrm{~nm}]$ and determined by the ratio of sodium citrate to gold starting material. ${ }^{7}$ Development of a more in-depth understanding of the potential nanotoxicity of these and other attractive therapeutic nanomaterials appears to depend on exploration of interactions of the many potential phospholipid-AuNP assemblies formed as a result of AuNP exposure.

Currently, citrate-stabilized AuNPs are used for multiple biomedical applications, including use as targeted drug carriers, thermal conductors for tumor ablation, and image enhancing agents. ${ }^{8}$ AuNP surface modification is considered easy due to functionalization 
permitting a wide range of biomolecules as ligands without compromising the integrity of the nanoparticle. ${ }^{9}$ Interactions of light with the nanostructure of AuNPs are used to harness photon energy to eradicate cancer cells using surface plasmon resonance. ${ }^{10}$ This oscillation is also responsible for the optical properties that attribute to the efficiency and intensity that gold nanoparticles can absorb and emit scattered light in biomedical imaging. ${ }^{11}$

Citrate-coated AuNPs have been observed in hyperspectral dark-field microscopy to integrate in between bilayers of dimyristoyl PC large unilamellar vesicles (LUVs) when AuNP diameter $=10[\mathrm{~nm}]$, disrupting integrity of the phospholipid vesicle. ${ }^{\mathbf{1 2}}$ It is beneficial to study a range of interactions of AuNP-phospholipid assemblies at the molecular level to further comprehension of physicochemical interactions and potential toxicological effects in future cancer nanotechnology applications. A study of interactions in the interior of the lipid membrane in this research was achieved by analysis frequency-domain lifetime and time-resolved fluorescence anisotropy decay measurements of AuNP-LUV assemblies.

The fundamental concept of fluorescence involves three steps. First, a susceptible molecule is excited by an incoming photon source, followed by vibrational relaxation of the excited state electrons to the lowest energy level. Finally, this phenomenon results in the emission of a photon of longer wavelength. Both frequency-domain lifetime and time-resolved fluorescence anisotropy decay measurements inform of how accessible the population of fluorescent probe molecule 1,6-diphenyl-1,3,5-hexatriene (DPH) embedded in a series of unsaturated phosphatidylcholine molecules will be to water by probing the membrane interior with light and electronically exciting DPH molecules embedded in the acyl chain region of the lipid bilayer. 
Fluorescence anisotropy is an analysis of the changing orientation of a susceptible molecule in a medium with respect to time between the events of emission and absorption of electromagnetic radiation. ${ }^{13}$ When the absorption event occurs, DPH retains polarization in the emission, and polarizers placed in the path of incident and emission rays of light can be analyzed by a fluorometer. In this research, absorption and emission events indicated alignment of the dipole of the DPH molecules relative to the wave of absorbed and emitted light.

\section{Purpose}

The objective of this research is to further the understanding of biomembrane-AuNP interactions and potential mechanisms of nanotoxicity in relation to biomedical applications of citrate-stabilized AuNPs through collection and interpretation of time-resolved fluorescence lifetime and anisotropy data resulting from experiments involving POPC exposure to citratecoated AuNPs. AuNPs of interest will range in diameter of 5, 10, and 20 [nm].

\section{Materials and Methods}

\subsection{Molecules of Interest}
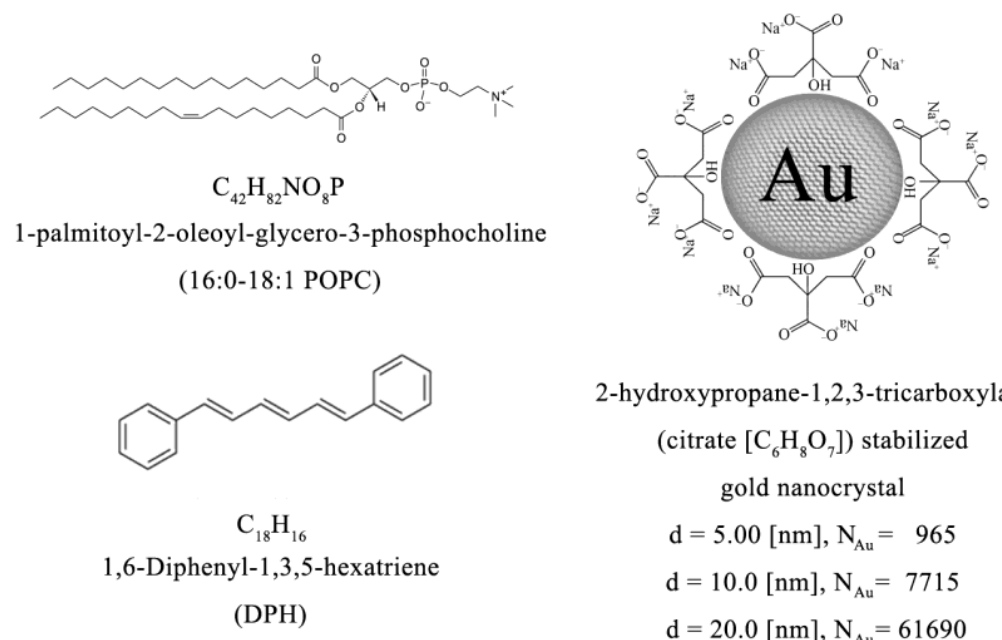

2-hydroxypropane-1,2,3-tricarboxylate (citrate $\left[\mathrm{C}_{6} \mathrm{H}_{8} \mathrm{O}_{7}\right]$ ) stabilized gold nanocrystal $\mathrm{d}=5.00[\mathrm{~nm}], \mathrm{N}_{\mathrm{Au}}=965$ $\mathrm{d}=10.0[\mathrm{~nm}], \mathrm{N}_{\mathrm{Au}}=7715$ $\mathrm{d}=20.0[\mathrm{~nm}], \mathrm{N}_{\mathrm{Au}}=61690$

Figure 1. POPC, DPH molecular structures and AuNP molecular representation (Note: ratio of citrate molecules to gold molecules varies per diameter of nanosphere. ${ }^{14}$ 


\subsection{Sample Preparation}

Freeze-thaw cycles $(8 \mathrm{x})$ were applied to sample of $2.0[\mathrm{~mL}, 8.1 \mathrm{mM}] 16: 0-18: 11$ palmitoyl-2-oleoyl-glycero-3-phosphocholine (POPC) stock solution purchased from Avanti Polar Lipids. POPC sample was stirred via vortexer for 10 seconds then 0.8 [mL] was extruded 11x through 0.2 [um] membrane filter paper to produce unilamellar lipid vesicles.

Three quartz cuvette samples were prepared for experimentation. Initially, each cuvette contained identical solutions composed of 1.2 [mL, $\mathrm{pH}$ 7.2] 4-(2-hydroxyethyl)-1piperazineethanesulfonic acid (HEPES) buffer with 0.022 [mL] extruded POPC LUVs to produce $0.15[\mathrm{mM}]$ POPC concentration. Following 10 seconds of manual agitation of POPC in HEPES buffer solution, 1.31 [uL] 1,6-diphenyl-1,3,5-hexatriene (DPH) dissolved in tetrahydrofuran (THF) (stored at $-20^{\circ} \mathrm{C}$ ) was additionally syringed into cuvette with solution to yield a phospholipid/DPH ratio of 300:1. All samples were brought to physiological temperature $37.0\left[{ }^{\circ} \mathrm{C}\right]$ via water bath.

After data was collected for control samples, each cuvette was designated for 5,10 and $20[\mathrm{~nm}]$ citrate-coated gold nanocrystals in water (concentration $0.04-0.05[\mathrm{mg} / \mathrm{mL}]$ ), purchased from NN-Labs. A low dosage range of 300.0 [uL] of each size AuNP was additionally pipetted into its designated cuvette for data collection at $37.0\left[{ }^{\circ} \mathrm{C}\right]$. For high dose range data set, each sample cuvette contained $37.0\left[{ }^{\circ} \mathrm{C}\right.$ ] solution composed of 900.0 [uL] AuNPs in water with $0.022[\mathrm{~mL}]$ extruded POPC LUVs, 1.2 [mL] HEPES buffer, and 1.31 [uL] DPH.

\subsection{Measurements and Analysis}

Time-resolved lifetime and anisotropy measurements of all samples were collected via Chronos digital frequency domain spectrofluorometer. Anisotropy decay intensity data was 
analyzed using a higher order mathematical model, known as the Brownian Rotational Diffusion model, in order to attain information regarding molecular disorganization. ${ }^{1}$

\section{Results}

In the following tables, outline average fluorescence lifetime $(\tau)$ measurements are provided along with average rotational correlation time to rotate one radian $(\phi)$. The rate of rotation of the dipole around the $\mathrm{z}$-axis propagating along the $\mathrm{X}$-axis is defined as $\boldsymbol{D}_{\perp}$. Furthermore, the disorder parameter based on $f(\theta)$ is identified in the tables below as $f_{\text {random }}$. The first set of data outline is in the $300.0[\mu \mathrm{L}]$ AuNP low dose range followed by data collected in the $900.0[\mu \mathrm{L}]$ AuNP high dose range.

Table 1. Low Dose Range:

POPC [0.15 mM] LUVs with $300.0[\mu \mathrm{L}] 0.04-0.05[\mathrm{mg} / \mathrm{mL}]$ citrate-stabilized AuNPs

\begin{tabular}{lcccccccc}
\hline & $\tau[\mathrm{ns}]$ & \pm & $\phi[\mathrm{ns}]$ & \pm & $\boldsymbol{D}_{\perp}[\mathrm{ns}]^{-1}$ & \pm & $\boldsymbol{f}_{\text {random }}$ & \pm \\
\hline POPC & 8.5 & 0.07 & 2.48 & 0.11 & 0.145 & 0.01 & 0.615 & 0.008 \\
$5 \mathrm{~nm}$ & 8.52 & 0.1 & 2.93 & 0.07 & 0.114 & 0.007 & 0.62 & 0.015 \\
$10 \mathrm{~nm}$ & 8.57 & 0.07 & 2.74 & 0.06 & 0.17 & 0.015 & 0.536 & 0.016 \\
$20 \mathrm{~nm}$ & 8.52 & 0.1 & 2.46 & 0.06 & 0.153 & 0.01 & 0.599 & 0.007 \\
\hline
\end{tabular}

Table 2. High Dose Range:

POPC $[0.15 \mathrm{mM}]$ LUVs with $900.0[\mu \mathrm{L}] 0.04-0.05[\mathrm{mg} / \mathrm{mL}]$ citrate-stabilized AuNPs

\begin{tabular}{lllllllll}
\hline & $\tau[\mathrm{ns}]$ & \pm & $\phi[\mathrm{ns}]$ & \pm & $\boldsymbol{D}_{\perp}[\mathrm{ns}]^{-1}$ & \pm & $\boldsymbol{f}_{\text {random }}$ & \pm \\
\hline POPC & 8.5 & 0.07 & 2.48 & 0.11 & 0.145 & 0.01 & 0.615 & 0.008 \\
$5 \mathrm{~nm}$ & 7.16 & 0.2 & 0.244 & 0.06 & 1.21 & 0.2 & 0.561 & 0.01 \\
$10 \mathrm{~nm}$ & 7.95 & 0.15 & 1.57 & 0.18 & 0.669 & 0.15 & 0.388 & 0.04 \\
$20 \mathrm{~nm}$ & 8.29 & 0.1 & 2.295 & 0.06 & 0.174 & 0.02 & 0.572 & 0.022 \\
\hline
\end{tabular}


Figures 2 and 3 display orientational probability distribution parameter $f(\theta)(\sin \theta)$ with respect to degrees from the membrane normal. The orientational distribution function requires the general constraints

$$
\begin{gathered}
f(\theta) \geq 0 \\
\int_{0}^{\pi} f(\theta)(\sin \theta) d \theta=1
\end{gathered}
$$

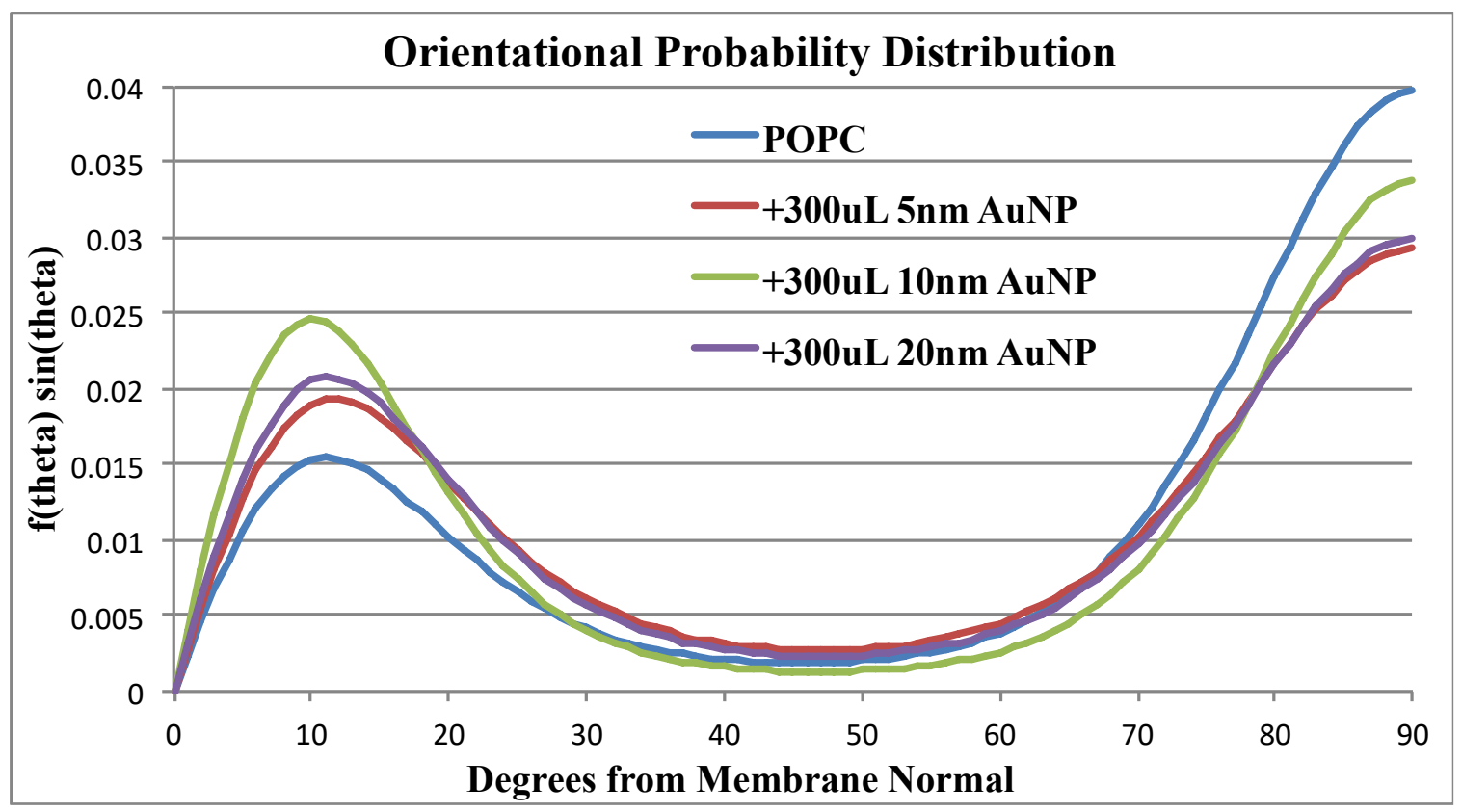

Figure 2. Orientational probability distribution of DPH probe in POPC bilayer with 300.0 [uL] $0.04-$ $0.05[\mathrm{mg} / \mathrm{mL}]$ citrate-stabilized AuNPs

Figures 2 and 3 display orientational probability distributions demonstrating the phospholipid acyl chains cause the fluorescent probe molecule DPH to be nonrandom. 


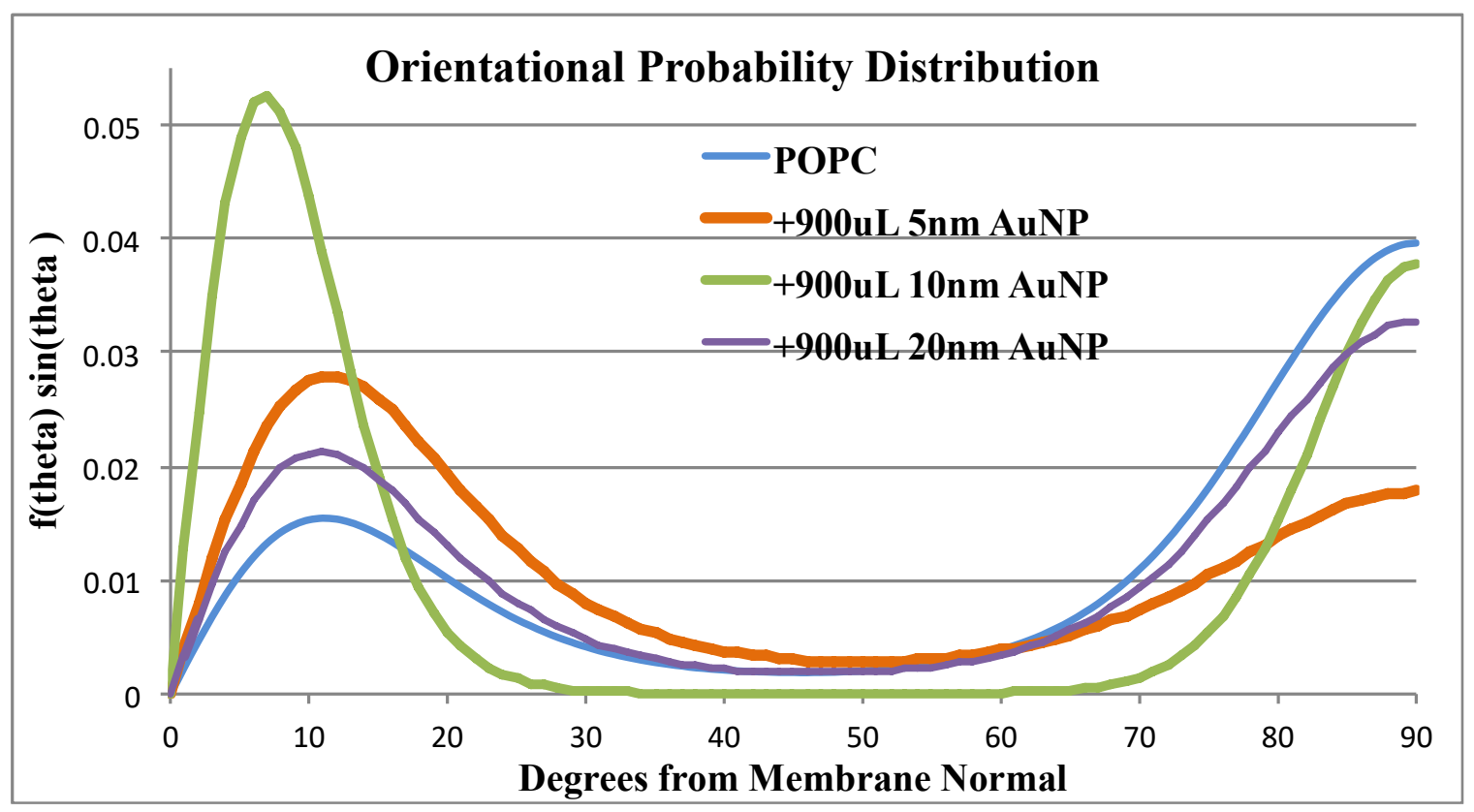

Figure 3. Orientational probability distribution of DPH probe in POPC bilayer with 900.0 [uL] $0.04-$ $0.05[\mathrm{mg} / \mathrm{mL}]$ citrate-stabilized AuNPs

If there existed a scenario where DPH would be examined in a completely random orientation, a disorder parameter exists where the probability distribution curve created under random conditions can be overlapped with the empirically derived orientational probability distributions as seen in Figure 4.

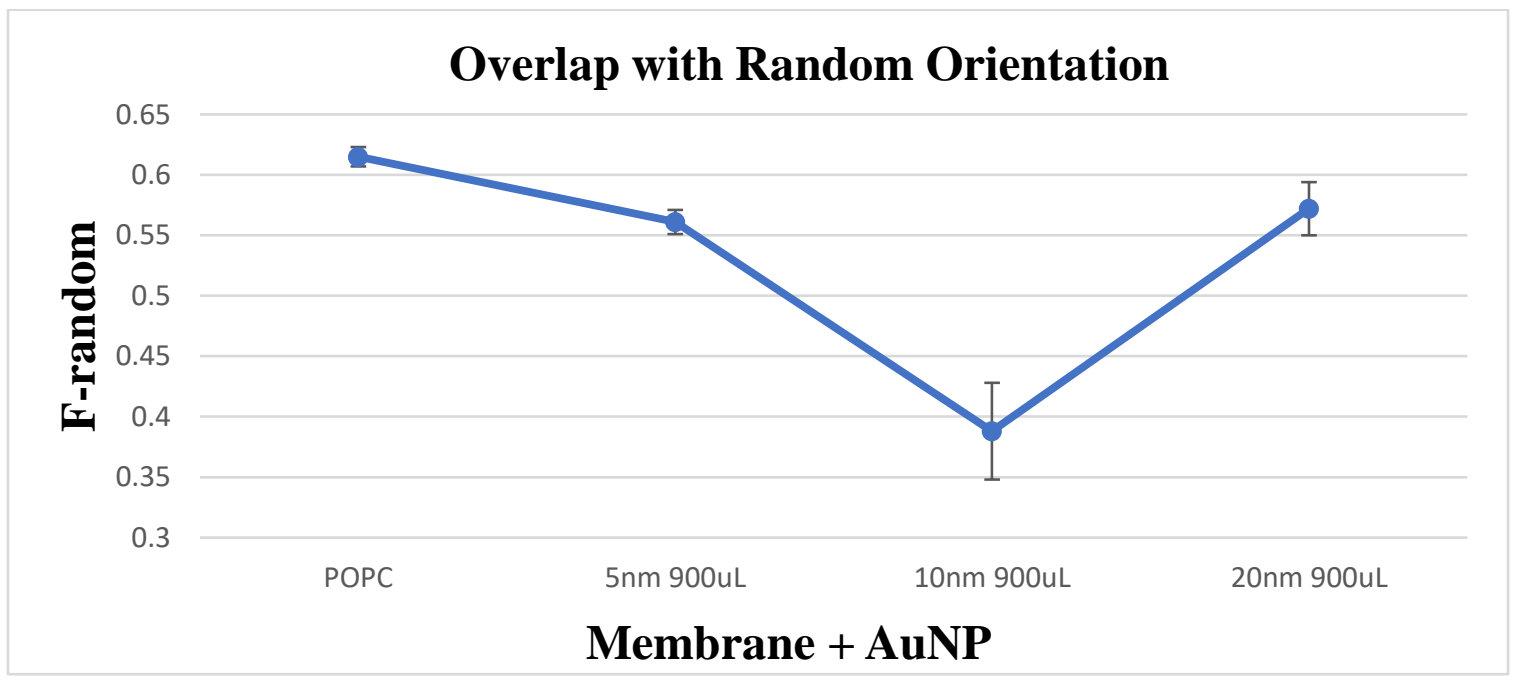

Figure 4. The probability of a random orientation overlapped with the orientational probability distribution displays the deviation displayed by the 10 [nm] AuNP samples. 
Frequency-domain lifetime measurements showed no significant change in fluorescence lifetime of DPH molecules in the administration of the low dose range of AuNPs (300.0 [uL] 0.04 $-0.05[\mathrm{mg} / \mathrm{mL}])$.

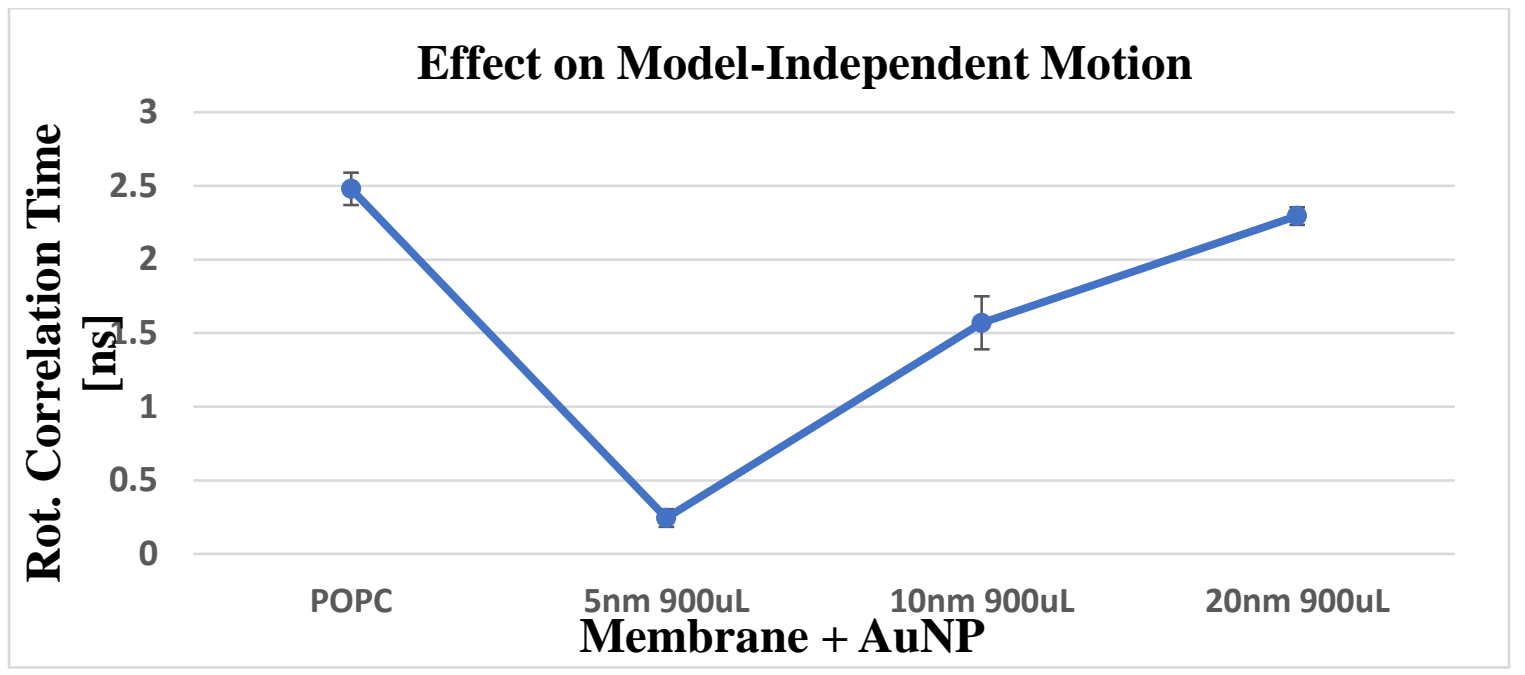

Figure 5. Rotational correlation lifetime of DPH probe in POPC bilayer for samples in high dose range

Fluorescence lifetime measurements were noteworthy in the high dose range of AuNPPOPC LUV assemblies (AuNP 900.0 [uL] $0.04-0.05[\mathrm{mg} / \mathrm{mL}]$ ).

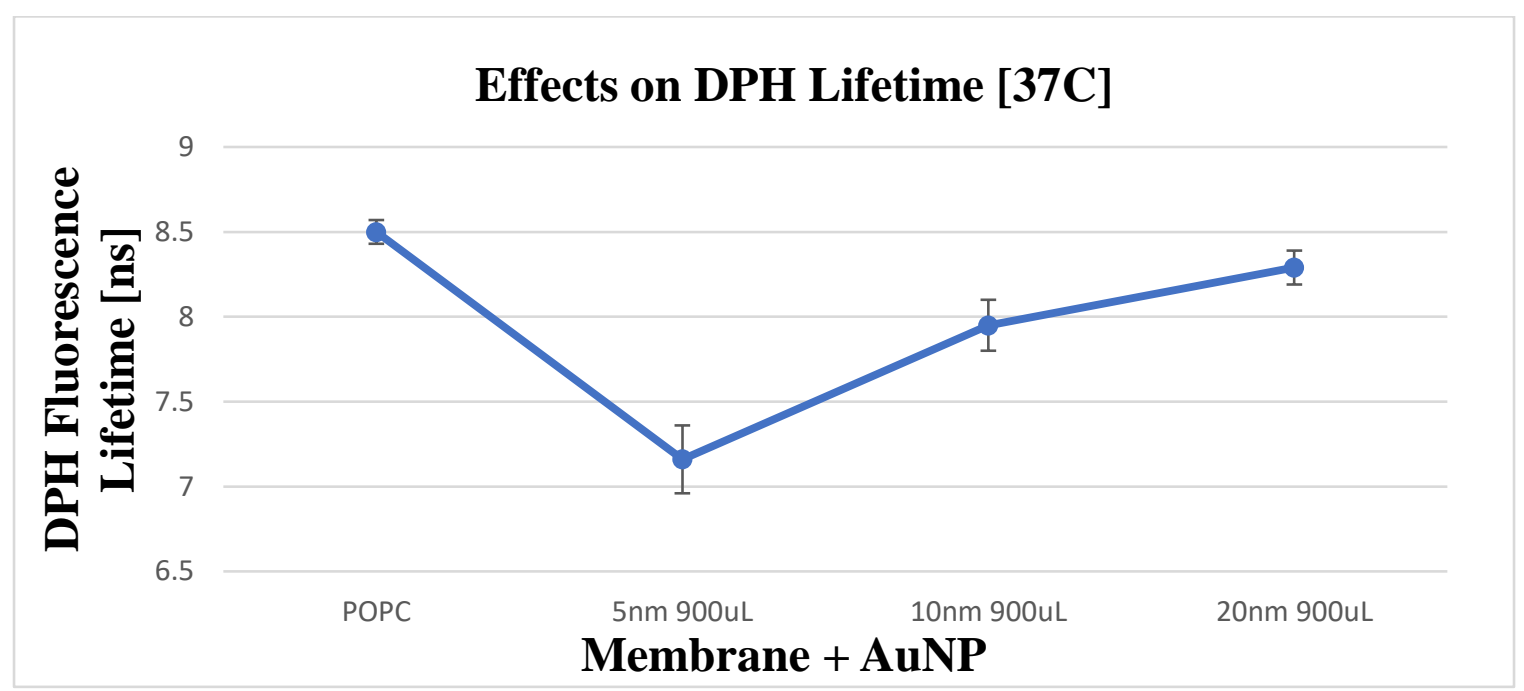

Figure 6. High-dose range fluorescent probe DPH lifetime before being exposed to water 


\section{Discussion}

The behavior of the DPH molecule was used to learn about acyl chain packing and subsequent structural properties in the POPC bilayers induced by exposure to citrate-capped AuNPs. In both low and high dosage ranges, including all size AuNPs, loss of density in packing of phospholipid head groups occurred due to increased water penetration into membrane. All sizes of AuNPs in both dosage ranges increased the rate of molecular rotational motion in the membrane bilayer. In the low dose range (300.0 [uL] citrate-stabilized AuNP in water) no significant change in fluorescence lifetime was identified. Slight variances were detected between $5[\mathrm{~nm}]$ diameter AuNPs and 10 [nm] size AuNPs, but the effects on POPC LUVs were near identical between $5[\mathrm{~nm}]$ and 20 [nm] diameter AuNPs in the low dose range. Suppression of orientations between two main populations was detected with 10 [nm] diameter AuNPs in low dose range.

Variances according to AuNP diameter were detected in samples of high dose range (900.0 [uL] citrate-capped AuNP in water). Evidence of rupture of POPC vesicles was observed when AuNP diameter equaled 5 [nm]. A more severe suppression of orientations between two main populations occurred when AuNP diameter equaled 10 [nm]. AuNPs with diameter of 20 [nm] exhibited a very small effect of restricting mobility of POPC LUVs.

The most notable demonstration of nanotoxicity with AuNP-POPC assemblies was in the high dose range when AuNP diameter was equal to 5 [nm]. The cause of vesicle lysis could potentially be due to chemical functionalization of POPC-AuNP assemblies or could be caused by decreasing the size of the nanoparticle to favor vesicle internalization. ${ }^{15}$ 
In summary, when 16:0 - 18:1 POPC unilamellar vesicles 200 [nm] diameter were dosed with $300.0[\mathrm{uL}] 0.04-0.05[\mathrm{mg} / \mathrm{mL}]$ citrate-coated AuNPs of 5, 10 and 20 [nm] diameter, an increase in POPC membrane rigidity was observed, but effects of all sizes of AuNPs in this dosage range expressed nominal effects on POPC membrane bilayer composition. POPC LUVs dosed with $900.0[\mathrm{uL}] 0.04-0.05[\mathrm{mg} / \mathrm{mL}]$ citrate-stabilized AuNPs showed more significant effects on lipid bilayers, including rupture of vesicles when AuNP diameter was equal to 5 [nm]. When AuNP diameter equaled 10 [nm], suppression of orientations between two main populations occurred. AuNP-LUV assemblies incorporating $20[\mathrm{~nm}]$ diameter AuNPs had a nominal effect on POPC when administered to LUVs in the high dose range.

An emergence of a range of biomedical applications of engineered nanomaterials and the potential risk of toxic effects makes it crucial to investigate and understand the physical chemistry of the interactions of biomembranes and nanoparticles ${ }^{16,17}$. Future research to determine the concentration threshold of citrate-capped AuNPs correlating with vesicle decomposition when exposed to 5 [nm] AuNPs will be beneficial documentation for biological applications where citrate-stabilized AuNPs are used to mediate cellular interventions. 


\section{Abbreviations}

AuNP: gold nanoparticle

LUV: large unilamellar vesicle

PC: phosphatidylcholine

DPH: 1,6-diphenyl-1,3,5-hexatriene

POPC: 1-palmitoyl-2-oleoyl-sn-glycero-3-phosphocholine

THF: tetrahydrofuran

HEPES: 4-(2-hydroxyethyl)-1-piperazineethanesulfonic acid 


\section{References}

1. Mitchell, D. (2018). Mapping the Interior of Membranes, Timing Fast Events in Vision, Plus Working at NIH. Portland State University Physics Society Faculty Talk, 16 Nov 2018.

2. Mitchell, D., Litman, B. (1998). Molecular Order and Dynamics in Bilayers Consisting of Highly Polyunsaturated Phospholipids. Biophysics Journal, Volume 74, Feb 1998, 879-891.

3. Reece, J. B., Urry, L. A., Cain, M. L. 1., Wasserman, S. A., Minorsky, P. V., Jackson, R., \& Campbell, N. A. (2014). Campbell biology (Tenth edition.). Boston: Pearson.

4. Chakraborty, S., Abbasi, A., Bothun, G., Nagao, M., Kitchens, C. (2018). Phospholipid Bilayer Softening Due to Hydrophobic Gold Nanoparticle Inclusions. Langmuir 2018, ACS Publications.

5. Mosquera, J., Henriksen-Lacey, M., Garcia, I., Martinez-Calvo, M., Rodriguez, J., Mascarenas, J., Liz-Marzan, L. (2017). Cellular Uptake of Gold Nanoparticles Triggered by Host-Guest Interactions. J. Am. Chem. Soc. 2018, 140, 4469-4472.

6. Hind Al-Johani et al, (2017). The structure and binding mode of citrate in the stabilization of gold nanoparticles, Nature Chemistry, 2018, vol. 9, 890-895.

7. Frens, G. (1973). Controlled nucleation for the regulation of the particle size in monodisperse gold suspensions. Nature: Phys Sci 241:20-22.

8. He, X., Lin, M., Li, F., Sha, B., Xu, F., Qu, Z., Wang, L. (2015). Advances in Studies of Nanoparticle-Biomembrane Interactions. Nanomedicine, 2015, 10(1), 121141.

9. Kong, F., Zhang, J., Li, R., Wang, Z., Wang, W., Wang, W. (2017). Unique Roles of Gold Nanoparticles in Drug Delivery, Targeting and Imaging Applications. Molecules, 22,1445, 31 August 2017.

10. Qian, H., Zhu, Y., Jin, R. (2012). Atomically precise gold nanocrystal molecules with surface plasmon resonance. PNAS January 17, 2012109 (3) 696-700.

11. Hu, S., Ren, Y., Wang, Y., Li, J., Qu, J., Liu, L., Ma, H., Tang, Y. (2019). Surface plasmon resonance enhancement of photoluminescence intensity and bioimaging application of gold nanorod CdSe/ZnS quantum dots. Beilstein J. Nanotechnol. 2019, 10, 22-31.

12. Bhat, A., Huan, K., Cooks, T., Boukari, H., Lu, Q. (2018). Probing Interactions between AuNPs/AgNPs and Giant Unilamellar Vesicles (GUVs) Using Hyperspectral Dark-field Microscopy. Int. J. Mol. Sci. 2018, 19(4), 1014.

13.Vogel, S., Thaler, C., Blank, P., Koushik, S. (2009). "Time Resolved Fluorescence Anisotropy." Microscopy in Biology and Medicine. Boca Raton: Taylor \& Francis, 245-290. 
14. Huang, R., Wen, Y., Shao, G., Zhu, Z., Sun, S. (2014). Single-crystalline and multipletwinned gold nanoparticles: an atomistic perspective on structural and thermal stabilities. Royal Society of Chemistry Advances: 15(24), 17 Jan 2014.

15. Fratoddi, I., Venditti, I., Cametti, C., Russo, M. (2015). How Toxic are Gold Nanoparticles? The State-of-the-Art Ilaria Fratoddil Dipartimento di Chimica, Universita' di Roma, Nano Research, June 2015, Vol 8(6), 1771-1799., 11 Dec 2014.

16. Zhang, X., Lopez, A., Liu, Y., Wang, F., Liu, J. (2018). Interactions between citrate-capped gold nanoparticles and polymersomes. Journal of Applied Physics Vol 51(24), 22 May 2018.

17. Kanduc, D.; Mittelman, A.; Serpico, R.; Sinigaglia, E.; Sinha, A. A.; Natale, C.; Santacroce, R.; Di Corcia, M. G.; Lucchese, A.; Dini, L.; Pani, P.; Santacroce, S.; Simone, S.; Bucci, R.; Farber, E. (2002). Cell death: apoptosis versus necrosis (review). Int. J. Oncology, 2002, 21, $165-170$. 